\title{
POST-OPERATIVE ENTERITIS
}

\author{
By Michael R. Williams, F.R.C.S.
}

Senior Lecturer in Surgery, Surgical Professorial Unit, St. Thomas's Hospital

Transient intestinal symptoms, such as colic, flatulence and diarrhoea, are common following any operation, particularly one in which the bowels are handled. This is due to disordered peristalsis of loops recovering from a period of inactivity. In the early stages of recovery the segmenting contractions of the small intestine predominate over the more highly organised propulsive waves and produce static collections of fluid (Devine, 1948). In some cases of recovering paralytic ileus and following vagotomy the diarrhoea is more marked and appears to be caused by multiplication of bacteria in these collections whose passage down the intestinal tract produces an acute irritation of the small bowel. Once the reservoir of infected fluid has been obliterated the latter tends to settle.

There is, however, another form of postoperation diarrhoea, which is of varying severity, but may occasionally be so catastrophic as to cause death within a few hours, and which may for want of a better name be known as necrotizing enteritis from the effect produced on the mucosa of the small intestine.

This condition, while not very common, may follow any operation, but more usually one on the intestines, in particular the stomach, and is a definite source of mortality and morbidity. Necrotizing enteritis has been described as an occasional complication for many years (Finney, 1893; Riedel, r902), but lately it has roused much more interest and large numbers of cases have been reported (Dixon and Weismann, 1948; Williams and Pullan, 1953; Terplan, 1953; Wakefield, 1953; Speare, 1954; Dawson-Edwards and Morrissey, 1955; Fowler, 1955). In fact it would appear that the condition is on the increase (Editorial F. Am. Med. Ass., I955). The opinion of Pettet et al. (1954) to the contrary is based only on post-mortem material and takes no account of surviving cases.

The clinical picture in a severe case is characteristic and extraordinarily constant.

A fit patient undergoes some such operation as partial gastrectomy, which is achieved without difficulty, or remarkable circumstance. For the first day or two his progress is satisfactory and he then complains of upper abdominal colic and appears anxious. His temperature may be a little raised but bowel sounds are present and his abdomen is soft, although possibly a little tender in the epigastrium.

In a few hours his skin becomes cold and clammy from peripheral constriction and the temperature may rise to 103 or $104^{\circ} \mathrm{F}$. He starts to pass thin, watery, offensive motions, sometimes bloodstained and occasionally containing shreds of a cast of the mucous membrane of his bowels. This may become an almost constant stream and be associated with vomiting of similar material. There is anuria.

The blood pressure falls and the pulse rises and, unless suitable treatment is started at once the patient may die in a few hours of peripherat vascular failure. Occasionally the onset is so acute that death intervenes before the diarrhoea occurs, in which case the abdomen is distended by the fluid in the lumen of the gut.

Cases appear to vary in severity from the catastrophic type just described through all gradations to fairly mild cases of post-operative diarrhoea with insufficient fluid loss to cause severe decrease of circulating volume and hence evidence of shock.

In mild cases, and in the severe ones tided over the acute phase by adequate treatment, resolution occurs over Io to I4 days during which the diarrhoea gradually lessens, with finally complete recovery.

The differential diagnosis in the severe cases is from leak at the suture line and peritonitis, paralytic ileus, haemorrhage, adrenal failure and other disasters such as coronary thrombosis and pulmonary embolism. These have undoubtedly caused great confusion and allowed cases to be missed both in the past and now (Hansboro, 1953). The presence of bowel sounds and the rising haematocrit (Weber, I955), with evidence of dehydration, should exclude the first three and awareness of the condition of necrotizing enteritis should help to prevent the diagnosis of the others in the absence of specific signs.

The post-mortem appearances of the rapidly fatal cases are extremely constant. 
The peritoneal cavity apart from a little free fluid is normal and there is no evidence of peritonitis. The suture lines, if there has been an anastomosis are intact and the outside of the intestines is normal, although if the case has survived for a few days there may be a little oedema of the bowel wall, with some injection of the visceral peritoneum. The gut may be distended with fluid.

On opening the bowel, however, it is found that over a varying area, usually mostly in the small intestine but also sometimes involving the stomach and large bowel, the mucous membrane is locally ulcerated, and a slough like a diphtheritic membrane has been formed. This latter is adherent to the mucous membrane in some places and elsewhere has become detached and been rolled into a bolus which is being passed down the gut, leaving the remnants of the mucous membrane bare and glazed. Such a cast has been passed per anum by cases that have recovered.

In many of the reported cases, especially the earlier series, if the stools have been cultured only selective media have been inoculated in a search for enteric organisms. These are never found. Usually there is little or no growth, or occasionally the plate is overgrown with E. Coli or Proteus, which is probably not significant.

If, however, a blood agar plate is used, a heavy growth of Staphylococcus aureus is frequently obtained, often in almost pure culture. In some cases positive blood cultures in vivo, or from heart and splenic blood post-mortem have been demonstrated (Wakefield and Sommers, 1953) showing that a bacteraemia of the staphylococci has occurred.

In all reports where sensitivities have been done, the organism is insensitive to the antibiotics with which the patient has been treated.

Other organisms are sometimes encountered, such as $\alpha$-Haemolytic (Williams and Pullan, r 953) or $\beta$ Haemolytic (Speare, I954) Streptococci.

Microscopically the slough consists of dead mucous membrane and fibrin, in which are embedded large numbers of bacteria. The remaining mucous membrane is partially necrotic, the process starting on the surface and extending to varying depths in various parts of the bowel. Almost everywhere some basal cells survive. The submucosa is oedematous and the small blood vessels are dilated but the cellular response is not marked and consists mostly of plasma cells and lymphocytes. The necrosis does not penetrate through into the submucosa or muscularis.

From these findings a rational treatment of the established acute case can be derived. The lesion of the small bowel is analagous to that of the skin in an extensive partial thickness burn. Due to the

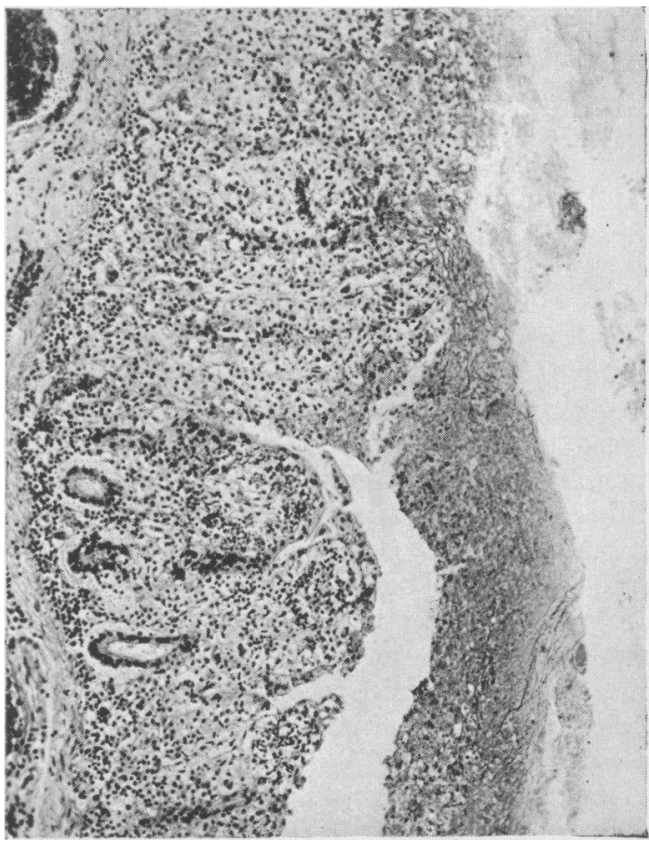

Section showing a partially separated slough with necrosis in the superficial parts of the mucosal glands and the survival of some basal portions. (Haematoxylin and eosin $x$ I I 2.)

loss of surface continuity, there is a massive outpouring of water and salts into the lumen of the bowel where they are lost to the body. But since the bases of the glands have survived, the lesion is essentially recoverable if the patient is kept alive until re-epithelialization has taken place.

The rapid loss of such vast quantities of fluid causes a decrease in the circulating volume in the blood vessels, thus producing the state of shock from which the patient dies.

Massive replacement of fluid intravenously must therefore be started at once. Clinical dehydration in an adult implies an extra cellular deficit of at least five litres, but vastly larger quantities, sometimes as much as 10 or 12 litres, may be required to restore hydration, the guide being the improvement in the patient's condition. Initally the fluid should be run in as quickly as possible, the only limit being a rising jugular venous pressure. To obtain a sufficiently rapid input, two or three drips running at the same time may be necessary (Hilsabeck and Dixon, I952; Williams and Pullan, 1953; Hayson, 1955). When hydration is achieved the blood pressure will rise, the peripheral spasm will relax, the patient become rational and a diuresis start. Since the great need is for water, the fluids given should be isotonic and it is best to give one litre of normal saline to 
two of 5 per cent. dextrose. Once hydration has been achieved the rate of infusion is slowed and only fresh losses are replaced.

In addition to water, there has been a loss of salts, proteins and red cells. The first are best replaced following estimation of the serum electrolytes after rehydration, when true estimates of requirements can be made without the confusion introduced by concentration.

Blood should be given usually during the convalescent period, although there is no objection to transfusion during resuscitation provided it is not allowed to slow down the rapid inflow of water, which is what the patient primarily needs.

Since in the majority of cases the condition is associated with a heavy growth of staphylococci in the intestines, it is probably logical to give an antibiotic. The cocci involved are usually insensitive to the more commonly used antibiotics, and therefore in this country erythromycin should be used until the sensitivities are available from the stool culture.

A nor adrenalin drip has been used (Jonassen, 1955) but this seems illogical as the patients are usually in intense peripheral spasm already.

Other authorities advise the administration of corticotropin. The three successful cases, however, reported by Prohaska (I954) do not seem to fall into the group under discussion, since in two they were still having severe diarrhoea on the thirtieth day, while in the cases observed by the author the condition had resolved in a fortnight.

Provided that the complication is diagnosed and adequate replacement of fluid is made, the recovery rate is high. Follow-up in surviving cases shows, as would be expected from the nature of the partial loss of the mucous membrane, a normal small bowel pattern in a barium meal and clinically no defect in absorption.

Any measure of prophylaxis implies a discussion of the aetiology. This is still obscure. The theory that it is due to anoxia and subsequent necrosis of the mucous membrane due to hypotension during the operation (Penner et al. 1939 and 1948), cannot be maintained since all recent observers confirm that in the majority of cases the operation went smoothly and there was no shock, until after the condition of necrotizing, enteritis

Post-operative necrotizing enteritis has occurred in both sexes and at all ages. In this hospital, the majority of cases have followed operations on the stomach, but we have seen it following operations on the colon, appendicectomy for acute appendicitis, hysterectomy and after a peripheral arterial graft. It has been reported following other operations not involving the abdomen such as radical mastectomy.
An analogous condition, with certain differences in the post-mortem appearances, probably due to the longer survival of the cases occurred spontaneously in mal nourished subjects in occupied Germany after the 1939-45 war (Fick and Wolken, 1949). This was said to be due to a toxin elaborated by colstridium Welchii Type $\mathrm{F}$ (Zeissler and Rassfeld-Sternberg, I949), but convincing confirmatory evidence is lacking.

It would also appear that in some of the cases of fulminating diarrhoea following intensive or prolonged widespectrum antibiotic therapy, similar pathological changes may occur. Such patients have passed a slough or cast of the mucous membrane which is similar to that seen in post-operative necrotizing enteritis, and the course of the disease is analagous (Nyphus, 1954; Williams, E., 1954). In cases coming to post-mortem the appearance may be indentical (Friedell and Paige, I954). In these cases it is found that the stools contain large numbers-often in almost pure culture, of staphylococci aureus which are resistant to the antibiotics with which the patient has been treated. In the earlier cases reported the organisms were penicillin resistant, but it is found that after a period of use of any new antibiotic resistant strains began to appear, and cases of staphylococcal enteritis have now been reported with strains resistant to all the commonly used antibiotics.

It has long been known that staphylococci car. elaborate an enterotoxin and that this is the cause of staphyloccal food poisoning. Although this causes a sharp bout of diarrhoea it is a mild disease and there is no post-mortem material available. In this condition the toxin is generated by the cocci while in the food and is ingested with it, the bacteria being inhibited by the other organisms present in the gut.

If the bowel has been sterilized, however, the cocci, having an undisputed field may continue to grow and thus produce a much larger dose of enterotoxin and hence more severe symptoms. That staphylocci occurring in cases of post antibiotic diarrhoea are capable of producing such an enterotoxin has been shown by Surgalla and Dack (1955). That post antibiotic enteritis is produced by the invasion of a sterilized gut by a resistant organism, which then multiplies and produces an enterotoxin which may at times cause lesions very similar to post-operative necrotizing enteritis, may now be accepted (Editorial Brit. med. F. 1955).

Unfortunately it is not possible to transfer this pathogenosis to the latter lock, stock and barrel for several reasons. The disease has been recorded for many years, long before antibiotics were available. Further, although in many casesespecially the earlier ones-the bacteriological 
investigations are inadequate, it has definitely been shown that the organism found is not always a staphylococcus, but may be a streptococcus. Further, although most of the recent cases in which the condition has occurred have received antibiotics pre or post operatively, or both, since this would now appear to be the practice in many clinics, several cases have appeared without any antibiotic medication (Williams and Pullan, I953).

The complication would, however, appear to be on the increase and sometimes appears in almost epidemic form. Six cases were encountered in two months in one ward at this hospital.

Following any operation involving an anesthetic, but particularly one in which the bowels are handled, there is a period of gastro-intestinal stasis. It is possible that under unfavourable circumstances bacteria may be introduced which are able to gain a hold and multiply, and if they produce a toxin may give rise to necrotizing enteritis. The flora of the small bowel is normally scanty, but this rapidly increases if there is any stasis. The Staphylococcus aureus is an unusual inhabitant of the small bowel except in the presence of achlorhydria (Barber and Franklin, 1946) but resistant forms are unfortunately common in the air of every hospital. It would therefore be easy for it to be introduced on a stomach tube, or by contaminated food or utensils, or indirectly from the patient's own throat or nose, which may become colonized during his stay in the ward. Usually the ingestion of a few staphylococci does not matter, even in the presence of achlorhydria, , since the competing bacteria ensure that they cannot propagate seriously during the period of stasis. However, some unfavourable circumstances may remove this inhibition and a major one would be the absence of other organisms, giving the pathogen an open field. Such an effect has been demonstrated in mice by Meynell.(I955) using a streptomycin resistant strain of Salm. Typhi-murium. The LD 50 of organisms introduced by a gastric tube was reduced from over a million in controls to less than five in animals prepared by previous oral streptomycin.

Dubos (1955) has shown that starvation of mice for 36 hours very greatly enhances the toxicity of various organisms, including Staphylococci aureus, when injected intravenously. But owing to the very high rate of metabolism of these small animals it seems unlikely that the normal preoperative period of starvation in man is at all comparable.

It would therefore appear that the routine preoperation preparation of patients with wide spectrum antibiotics is unwise and should be reserved for cases in which there is some special and over-riding indication. If possible only drugs with a restricted field of action should be used, such as the sulphonamides. Elective procedures on patients whose nose or throak contain coagulase positive staphylococci should if possible be postponed. And if pre-operative. antibiotics are essential steps should be taken tक्F lessen the hazard of ingestion of pathogenic bacteria.

\section{Summary and Conclusion}

Post-operative necrotizing enteritis is an acute and catastrophic condition with a characteristices clinical pattern, which must be distinguished fromother post-operative disasters. If diagnosed earlyand treated energetically the majority of cases make $\vec{\omega}$ a complete and lasting recovery without sequelaes

The complication is on the increase, and this iso probably due to the misguided use of wide spectrum antibiotics, which sterilize the gut and allow the multiplication of resistant staphylococctiv which elaborate an enterotoxin which produces the characteristic lesion. Care throughout should be exercised in the use of widespectrum antibiotics before or during a period of intestinal stasis.

My thanks are due to Dr. H. Spencer, M.D. $\vec{e}$ for the illustration and to Professor J. B $\mathbb{\Phi}$ Kinmonth, M.S., and Mr. J. M. Pullan, M.Ch. for their interest.

\section{BIBLIOGRAPHY}

BARBER, M., and FRANKLIN, R. H. (r946), Brit. med. F., i, 迎 $\overrightarrow{0}$

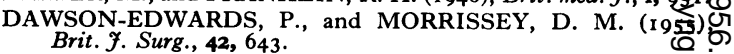

DEVINE, J. (1946), Ibid., 34, 158.

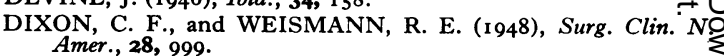

DUBOS, R. J. (1955), Proc. Roy. Soc. Med., 48, 911.

EDITORIAL (1955), Brit. med. ., i, 1328.

EDITORIAL (1955), f. Amer. Med. Ass., 157, 346.

FICK, K. A., and WOLKEN, A. P. (1949), Lancet, i, 519.

FINNEY, J. M. (1893), Bull. fohns Hopk. Hosp., 4, 53.

FOWLER, B. J. (1955), Brit. med. F., i, 1313.

FRIEDELL, G. H., and PAIGE, E. (1954), Amer. F. Clin. Path. 24, 1159.

HANSBORO, G. L. (1953), Amer. Surg., 19, 82.

HAYSOM, A. H. (1955), Brit. med. F., ii, 57.

HILSABECK, J. R., and DIXON, C. F. (1952), Amer. F. Surg. 84, II4.

JONASSEN, O. T., et al. (1955), New Eng. Ұ. Med., 252, 792.

MEYNELL, G. G. (1955), Proc. Roy. Soc. Med., 48, 916.

NYPHUS, R. E., and BURKE, E. C. (1954), Proc. Mavo Clin. 29, 513 .

PENNER, A., and BERNHEIM, A. I. (1939), Arch. Path., 27, 966 극 PENNER, A., and DRUCKERMAN, L. J. (1948), Gastroenterology II, 478 .

PETTET, J. D., et al. (1954), Surg. Gynec. Obstet., 98, 546.

PROHASKA, J. V., et al. (1954), Э. Amer. Med. Ass., 154, 320. $\frac{D}{O}$ RIEDEL, B. M. C. L. (1902), Dtsch. Z. Chir., 67, 402, cited by․ㅡ. Penner and Bernheim (1939).

SPEARE, G. S. (1954), Amer. F. Surg., 88, 523.

SURGALLA, M. J., and DACK, G. M. (1955), f. Amer. med. Ass" I58, 649 .

TERPLAN, K., et al. (1953), Gastroenterology, 24, 476. WAKEFIELD, R. D., and SOMMERS, S. C. (1953), Ann. Surg.

WEBER, B. M. (1955), N. Y. St. F. Med., 55, 2383.

WILLIAMS, E. (1954), Lancet, ii, 999.

WILLIAMS, M. R., and PULLAN, J. M. (1953), Ibid., ii, ror3.

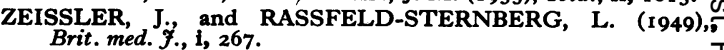

